\title{
вмJ Global Health Effects of PACK guide training on the management of asthma and chronic obstructive pulmonary disease by primary care clinicians: a pragmatic cluster randomised controlled trial in Florianópolis, Brazil
}

\author{
Max Oscar Bachmann (D) , ${ }^{1}$ Eric D Bateman, ${ }^{2}$ Rafael Stelmach, ${ }^{3}$ Alvaro A Cruz (D) ,4 \\ Matheus Pacheco de Andrade, ${ }^{5}$ Ronaldo Zonta, ${ }^{5}$ Jorge Zepeda, ${ }^{6}$ Sonia Natal, ${ }^{7}$ \\ Ruth Vania Cornick, ${ }^{2}$ Camilla Wattrus (D) ${ }^{2}$ Lauren Anderson, ${ }^{2}$ \\ Daniella Georgeu-Pepper, ${ }^{2}$ Carl Lombard, ${ }^{8}$ Lara R Fairall ${ }^{2}$
}

To cite: Bachmann MO, Bateman ED, Stelmach R, et al. Effects of PACK guide training on the management of asthma and chronic obstructive pulmonary disease by primary care clinicians: a pragmatic cluster randomised controlled trial in Florianópolis Brazil. BMJ Global Health 2019;4:e001921. doi:10.1136/ bmjgh-2019-001921

Handling editor Seye Abimbola

Received 15 August 2019 Revised 12 November 2019 Accepted 17 November 2019

Check for updates

(c) Author(s) (or their employer(s)) 2019. Re-use permitted under CC BY-NC. No commercial re-use. See rights and permissions. Published by BMJ.

For numbered affiliations see end of article.

Correspondence to Professor Max Oscar Bachmann M.Bachmann@uea.ac.uk

\section{ABSTRACT}

Introduction The Practical Approach to Care Kit (PACK) guide was localised for Brazil, where primary care doctors and nurses were trained to use it.

Methods Twenty-four municipal clinics in Florianópolis were randomly allocated to receive outreach training and the guide, and 24 were allocated to receive only the guide. 6666 adult patients with asthma or chronic obstructive pulmonary disease (COPD) were enrolled, and trial outcomes were measured over 12 months, using electronic medical records. The primary outcomes were composite scores of treatment changes and spirometry, and new asthma and COPD diagnosis rates.

Results Asthma scores in 2437 intervention group participants were higher $(74.8 \%, 20.4 \%$ and $4.8 \%$ with scores of 0,1 and 2, respectively) than in 2633 control group participants $(80.0 \%, 16.8 \%$ and $3.2 \%$ ) (OR for higher score $1.32,95 \% \mathrm{Cl} 1.08$ to $1.61, \mathrm{p}=0.006$ ). Adjusted for asthma scores recorded in each clinic before training started, the OR was 1.24 (95\% Cl 1.03 to $1.50, p=0.022$ ). COPD scores in 1371 intervention group participants (77.7\%, $17.9 \%$ and $4.3 \%$ with scores of 0,1 and 2 ) did not differ from those in 1181 control group participants (80.5\%, 15.8\% and 3.7\%) (OR 1.21, 95\% Cl 0.94 to 1.55 , $\mathrm{p}=0.142$ ). Rates of new asthma and COPD diagnoses, and hospital admission, and indicators of investigation, diagnosis and treatment of comorbid cardiovascular disease, diabetes and depression, and tobacco cessation did not differ between trial arms.

Conclusion PACK training increased guideline-based treatment and spirometry for asthma but did not affect COPD or comorbid conditions, or diagnosis rates. Trial registration NCT02786030 (https://clinicaltrials.gov/).

\section{INTRODUCTION}

Asthma and chronic obstructive pulmonary disease (COPD) are major contributors to

\section{Summary box}

What is already known?

- Educational outreach to primary care nurses, to improve diagnosis and treatment of adult chronic disease with clinical guidance, has been proven effective in South Africa.

What are the new findings?

- Similar outreach education to primary care nurses and doctors in Florianópolis, Brazil, increased initiation and alteration of asthma treatments, and use of spirometry for asthma, but did not change chronic obstructive pulmonary disease (COPD) management or rates of asthma and COPD diagnosis.

What do the new findings imply?

- Practical Approach to Care Kit training is an effective method of improving management of adult asthma in this urban Brazilian setting.

the burden of chronic illness in Brazil. The Global Burden of Disease Study 2016 ranked COPD as the eighth highest disease in Brazil in terms of disability-adjusted life years lost. ${ }^{1}$ Asthma was ranked $17 \mathrm{th}$ in terms of years lived with disability. ${ }^{1}$ Despite progress in tobacco control, in 2016 tobacco use was the fifth most important cause of disability-adjusted life years lost, especially through its effects on chronic respiratory disease. ${ }^{1}$ Epidemiological studies have found high rates of undiagnosed and untreated or inappropriately treated asthma and COPD in Brazil. ${ }^{23}$ WHO's World Health Survey found that $23 \%$ of Brazilian adults aged 18 to 45 years reported wheezing 
in the last year, of whom only $12 \%$ had a doctor diagnosis of asthma. ${ }^{3}$ Comorbidity is increasing, ${ }^{4}$ with high and growing rates of chronic conditions like cardiovascular disease, diabetes and depression.

Provision of good-quality primary healthcare, including investigation, diagnosis and appropriate treatment of asthma, COPD and comorbid conditions, is a crucial part of the solution. Since 1998, Brazil increased provision of free primary care by municipalities through the Family Health Programme of the national Unified Health System. ${ }^{5}$ Brazilian municipalities that provided free inhaled corticosteroids (ICSs) for asthma experienced larger reductions in hospital admissions and deaths from asthma than those who did not, ${ }^{6}$ and those who provided more long-acting beta ${ }_{2}$ agonists (LABAs) had larger reductions in hospital admission rates for COPD. ${ }^{7}$ Programmes to improve asthma care in two cities, with physician education, and free ICS and LABA, also reduced hospital admissions. ${ }^{89}$

The present study evaluates an initiative to improve integrated primary care including for asthma, COPD and comorbid chronic conditions across a Brazilian city by adapting a programme that has been developed and delivered at scale in South Africa. The Practical Approach to Care Kit (PACK) programme was developed by the Knowledge Translation Unit (KTU) of the University of Cape Town Lung Institute, in collaboration with government health departments in South Africa. ${ }^{10}{ }^{11} \mathrm{It}$ has four components-a clinical decision support tool or guide, primary care worker training, health system strengthening, and monitoring and evaluation. ${ }^{12}{ }^{13}$ PACK has been localised for, and is being used in, Nigeria, ${ }^{14}$ Ethiopia ${ }^{15}$ and Botswana. ${ }^{16}$

PACK was localised to the Brazilian context, and specifically for municipal primary healthcare clinics in the city of Florianópolis, between 2014 and 2016, with leadership by local primary care doctors mentored by the KTU. ${ }^{17}$ Florianópolis has a population of 486000 and was the first Brazilian municipality to provide universal health coverage under the auspices of the national Family Health Strategy. Although it is one of the wealthiest cities in Brazil, with a large private health sector, a substantial proportion of its population has lower incomes and depends on free municipal primary care facilities. There are 48 municipal facilities, with ambulatory care delivered by pairs of family doctors and nurses, between one and seven (median 2.5) doctor-nurse pairs in each, and 750-8500 (median 2700) adults served by each facility. Both nurses and doctors manage adults with respiratory symptoms, but nurses are not authorised to prescribe corticosteroids or beta ${ }_{2}$ agonists, referring patients with asthma or COPD to doctors for initiation or changes of treatments.

The purpose of using PACK in Florianópolis was to improve the quality and equity of primary healthcare for adults, while expanding and redefining the clinical roles of doctors and nurses. Before the study, the Florianópolis city health department identified asthma, diabetes, hypertension, back pain and tuberculosis as priorities for quality improvement, noting, for example, that in 2016, over $60 \%$ of adults diagnosed with asthma had never received inhaled corticosteroids, $20 \%$ had also been diagnosed with COPD, 38\% with hypertension or cardiovascular disease, and $12 \%$ with diabetes. Therefore, PACK's emphasis on integrated diagnosis and management of multiple conditions was deemed appropriate. PACK training covered multiple conditions, but the current study focuses only on its effects on participants with asthma and COPD.

The objective of the trial was to evaluate the effects of PACK training on the diagnosis, investigation and treatment of asthma and COPD in adults attending Florianópolis municipal clinics. A parallel trial of the impact of the same PACK training on cardiovascular and diabetes care, including using PACK to expand the scope of nurses, was planned and is in analysis. ${ }^{18}$ This paper reports the impact of PACK educational outreach training on primary care of respiratory conditions during the first year after active implementation and is part of a Collection of papers on PACK.

\section{METHODS}

\section{Trial design}

This was a pragmatic, parallel-group, superiority cluster randomised $\operatorname{trial}^{18}$ (figure 1 and figure 2). All but one of 49 municipal clinics in Florianópolis were included in the trial; a small mobile clinic was excluded. Outcomes were measured over two baseline and one follow-up period (figure 1). The follow-up period was the 12 months after initial training ended (figure 1). The first baseline period, defined in the protocol, ${ }^{18}$ was the 12 months before follow-up began, during which initial training was completed (baseline 1 in figure 1). The second and earlier baseline period, adopted during the conduct of the study, was the 12 months before training began (baseline 2 in figure 1). Baseline 1 was originally specified because we assumed that the intervention would have no effect before initial training had been completed. ${ }^{18}$ However, trainers observed that delivery of training was associated with immediate effects on asthma and COPD care, which was likely to bias participants' baseline characteristics, and therefore also the estimates of effectiveness during follow-up. We therefore defined the second baseline period before training began (baseline 2). The follow-up period was 1 April 2017 to 31 March 2018 (figure 1). Baseline 1 period was 1 April 2016 to $31 \mathrm{March}$ 2017. Baseline 2 period was 1 July 2015 to 30 June 2016.

\section{Patient and public involvement}

Patients and public were not involved in carrying out this study. However, Brazilian health professionals and health professional organisations were consulted in developing the PACK guide.

\section{Randomisation}

Clinics were grouped into six strata, defined by numbers of doctor-nurse teams in each clinic (1-2, 3-4 teams or 


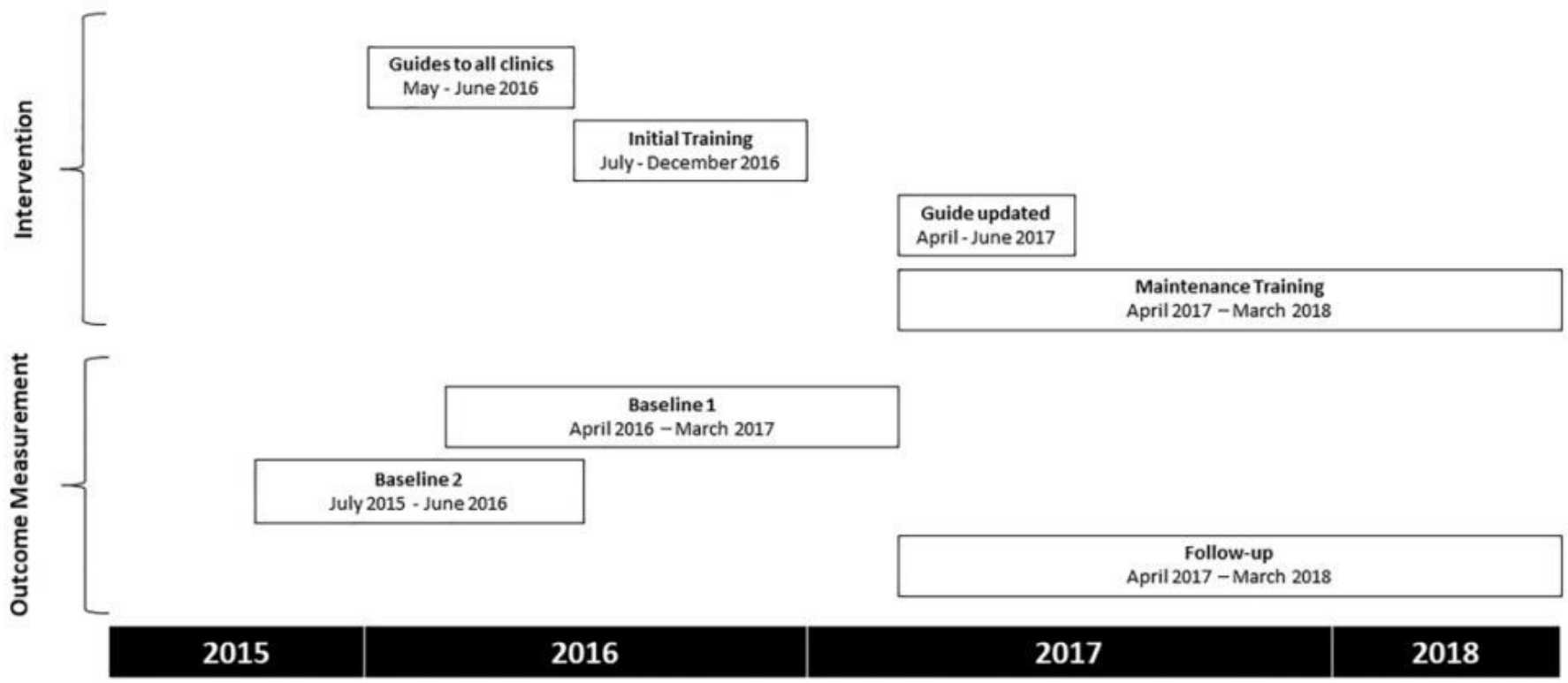

Figure 1 Timing of interventions, data collection and follow-up periods.

$\geq 5$ teams), and by their geographical location, which served as an indicator of socioeconomic position. A trial statistician (CL) randomly allocated clinics within each stratum to intervention and control arms in a 1:1 ratio using nQuery Advisor before the intervention began.

\section{Blinding}

Outcomes were extracted from routine electronic medical records and so recorded without participants' input, thus blinding of patients was not necessary. Blinding of health professionals was not possible because of the nature of the intervention.

\section{Study populations}

Eligible participants were identified using International Classification of Disease (ICD-10) diagnostic codes in electronic medical records for each clinic visit, in a consolidated municipal database. Patients included were all those aged 18 years and over in March 2017, who attended a participating clinic during the first year of the follow-up (figure 2), with a clinical diagnosis of obstructive lung disease (ICD-10 codes 40-47) recorded in electronic medical records since 1 January 2010, when electronic medical recording began. This study population was subdivided into those with asthma (with or without COPD) and those with COPD (with or without asthma). An additional study population comprised all adults who attended participating clinics during 12 months after training ended, in whom the annual rates of new diagnoses of asthma and COPD were estimated (figure 2). No sampling was used.

\section{Delivery of PACK in intervention and control group clinics}

Both intervention and control clinics were provided with the PACK guide in May and June 2016, and were included in the health systems strengthening, and monitoring and evaluation components of PACK, ${ }^{17}$ but only intervention clinics received training during the period covered by this study. The trial therefore evaluates the effect of PACK training.

The PACK guide is an integrated, comprehensive clinical decision support tool for use during primary healthcare consultations. ${ }^{12}$ A global version of the guide (freely available at https://knowledgetranslation.co.za/ pack/) provides an evidence-aligned and WHO guidance-aligned clinical approach to common symptoms, including cough, difficulty breathing, wheeze and chest pain in its first half and the remainder covers chronic conditions, including asthma, COPD, tuberculosis, cardiovascular disease and risk, diabetes and depression, and health promotion. The chronic respiratory disease section covers investigation, diagnosis and treatment of asthma and COPD, use of inhalers, spacers and peak flow meters. The Florianópolis version, comprising 103 pages, was provided in printed hard copy (from May 2016 onwards) and as an interactive PDF during the late stage of data collection (from November 2017 onward). ${ }^{17}$ This version of the guide, PACK Brasil Adulto-versão Florianópolis, was adapted to fit local needs and resources, and 49 local and national guidelines and protocols, and translated into Brazilian Portuguese. ${ }^{17}$ Local primary care professionals, medical specialists, health service managers and other stakeholders were involved in localisation. Unique features of the Florianópolis adaptation included the first use of a mentorship model to support localisation, ${ }^{10}$ guidance on smoking cessation, and expansion of PACK's colour coding system to reflect nurse and doctor role definitions.

PACK training comprised educational outreach for primary care doctors and nurses on how to use the PACK guide, using clinical case scenarios. ${ }^{12}$ Training was provided in or near the primary care facilities where trainees worked, entailed short interactive group sessions 


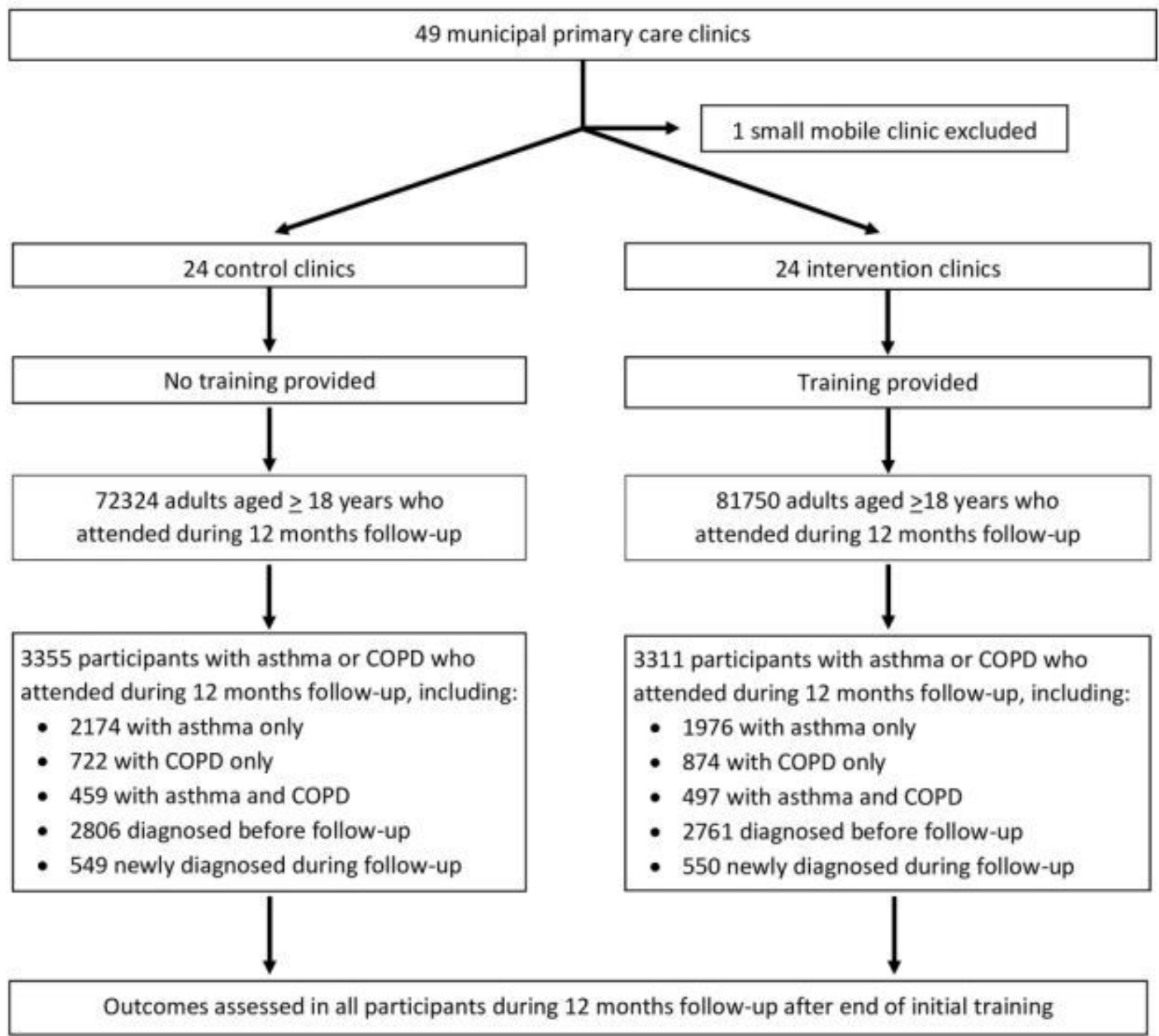

Figure 2 CONSORT diagram of recruitment and follow-up in intervention and control clinics. COPD, chronic obstructive pulmonary disease.

of about 90 min which were repeated about weekly during initial training and fortnightly during maintenance training. Training sessions were led by facility trainers who worked in the same facility or within the local health system. More detail on the training model is provided in another paper in this Collection. ${ }^{13}$ Unique features of the training in Florianópolis included appointing interprofessional nurse-doctor pairs to deliver outreach sessions, embedding master trainers, responsible for training and supporting the facility trainers, within the Primary Healthcare Department, and a curriculum focused on locally identified priorities, including respiratory conditions, diabetes, hypertension, back pain and tuberculosis. Initial training in intervention clinics took place during 12 sessions over 6 months (figure 1). After a pause of 3 months, 14 maintenance training sessions were delivered over 12 months. Training was delivered to 160 doctors and nurses, all of whose responsibilities included asthma and COPD care. Three municipal strikes within the context of political upheaval interrupted smooth implementation. ${ }^{17}$ After initial training, the pairs of facility trainers visited each clinic monthly, and the master trainers continued to communicate with facility trainers using email and a WhatsApp group.

\section{Primary outcomes}

There were three primary outcomes. The first two represent key healthcare processes that in Brazil and elsewhere have been shown to be associated with better health outcomes in patients with asthma and COPD, reflect the quality and intensity of respiratory care, and are based on actions recommended in the PACK guide. ${ }^{6-9} 1920$ The third primary outcome addresses the problem of underdiagnosis. ${ }^{23}$

1. For participants with asthma the composite score comprised points awarded for (a) first prescription of an ICS, or ICS+LABA combination (LABA+ICS), or a change in prescription: stepping up from short-acting 
beta $_{2}$ agonist (SABA) to ICS or from ICS to LABA+ICS; or stepping down from LABA+ICS to ICS, or from ICS to SABA (scoring one point if at least one of these occurred); and (b) request for spirometry (one point). The asthma score is the sum of these points, and ranges from 0 to 2.

2. For participants with COPD, the composite score comprised points awarded for (a) a first prescription of SABA, ICS or ICS+LABA; or a change in prescription, stepping up from SABA to LABA or from LABA to ICS+LABA, or stepping down from LABA+ICS to LABA, or from LABA to SABA (scoring one point if at least one of these occurred) and (b) request for spirometry (one point). The COPD score is the sum of these points, and ranges from 0 to 2.

3. At clinic level, the diagnosis rate was defined as the ratio between numbers of new diagnoses of asthma or COPD in patients aged 18 years and over to the total numbers of patients aged 18 years and over attending each clinic during baseline and 12-month follow-up periods.

\section{Secondary outcomes}

Secondary outcomes were the disaggregated treatment and spirometry components of asthma and COPD scores, prescriptions to support tobacco cessation (nicotine replacement therapy, nortriptyline or bupropion), cardiovascular disease (ICD-10 I00-I99) diagnosed for the first time, diabetes mellitus (ICD-10 E10-E14) diagnosed for the first time, cardiovascular risk assessed (ie, blood pressure recorded, or cholesterol, glucose or ECG tests requested), depression (ICD-10 F32-F34) diagnosed for the first time and medication for depression (tricyclic and related antidepressants, selective serotonin re-uptake inhibitors or monoamine oxidase inhibitors) prescribed for the first time. Outcomes relating to cardiovascular disease, diabetes and depression were indicators of awareness and management of potential comorbidity in people with chronic respiratory disease. Clinical level ratios between numbers of hospital admissions for asthma or COPD, and total numbers of patients aged $\geq 18$ attending each clinic during baseline and follow-up periods, were respiratory health indicators.

\section{Data collection and management}

Quantitative data on outcomes and baseline variables were extracted from the municipal health department's electronic medical records. Clinical data (including coded symptoms, ICD-10 coded diagnoses, prescriptions and test requests) were routinely entered during each consultation by a doctor or nurse, and linked at city, clinic and patient levels. The database was actively managed and regularly interrogated by a member of the Primary Healthcare Department (MPdA).

\section{Sample size and power}

The sample size calculation for patients with asthma assumed, based on previous medical records, that about
2900 eligible patients with asthma would attend 48 participating clinics during the year of follow-up, with mean asthma scores of 0.43 (SD 0.50) in the control group and intra-clinic correlation (ICC) of 0.033. The sample size calculation for patients with COPD assumed that about 1400 eligible patients with COPD would attend participating clinics during the year of follow-up, with mean COPD scores 0.42 (SD 0.48) in control clinics and ICC of 0.055 . This would provide $90 \%$ power to detect a $26 \%$ increase in mean asthma score (0.54 vs 0.43$)$, and a $33 \%$ increase in mean COPD score (0.56 vs 0.42), with $5 \%$ significance. At clinic level, the mean annual rate of new diagnoses of asthma or COPD was 11 (SD 4.6) per 1000 patients aged $\geq 18$ attending for any reason, providing $85 \%$ power to detect a $36 \%$ increase in rates of diagnosis (15 vs 11 per 1000 per year). With Bonferroni correction to the significance level to account for having three primary outcomes $(\mathrm{p}=0.05 / 3=0.017)$, the power to detect these differences as significant at the $1.7 \%$ level was $84 \%$, $82 \%$ and $73 \%$, respectively.

\section{Statistical analysis}

Statistical analyses were conducted to estimate the magnitude of effects by comparing outcomes between intervention and control groups, using Stata V.15 statistical software. Diagnosis rates in each clinic were compared at clinic level using linear regression. All other outcomes were compared at individual patient level, using regression models with robust adjustment for intra-cluster correlation of the outcome by clinic. Ordinal logistic regression was used for analyses with asthma and COPD scores as outcomes. Logistic regression was used for all other, binary, outcomes. Three sets of all analyses were carried out, first without adjustment for baseline values of the respective outcome, second with adjustment for baseline values recorded during baseline 1 and third with adjustment for baseline values recorded during baseline 2 (figure 1). The baseline values used for adjustment were mean values recorded in each clinic, instead of individual level values, so as to be able to include participants newly diagnosed during the follow-up period. All regression models included randomisation stratum as a covariate. Intra-cluster correlation coefficient for each outcome variable was estimated using one-way analysis of variance. We carried out subgroup analyses, to test whether effects differed between participants with newly or previously diagnosed asthma or COPD, by adding arm-subgroup interaction terms to the regression models. We also tested whether effects differed between subgroups of participants with both asthma and COPD and those with only asthma or COPD.

\section{Ethics and research governance}

Ethical guidance on cluster randomised trials and on use of medical records for research was adhered to. ${ }^{21} 22$ Identification of eligible participants and outcome measurement used electronic medical records without studyspecific patient contact. It was not feasible to obtain 
patients' consent to be randomised to intervention or control arms because randomisation and delivery of the intervention were at clinic level.

Patients were not asked for consent for their electronic medical records to be used for this research because it was not feasible. However, we adhered to the ethical principles for use of medical records without patients' consent, ${ }^{22}$ as follows. The research had a clear public benefit. We obtained approval for the study from the lead doctors and nurses managing the programme. Use of the data for research did not influence decisions about individuals' care. Only health department data managers had access to personal identifiers. Data with patient identifiers were held by the Florianópolis City Health Department as part of routine data management, and anonymised unlinked data were securely stored and analysed at the University of East Anglia.

\section{RESULTS}

The trial participants comprised 6666 adults with a new or previous diagnosis of asthma or COPD who attended a participating clinic for any reason during 12 months after the end of initial training (figure 2). The median number of visits per participant during this period was 5 (IQR 2-9). The median number of participants in each control clinic was 115 (range, 34-504) and the median number in each intervention clinic was 133 (range, 43-364). Of the 6666 participants, 3355 (2633 with asthma and 1181 with COPD, including 459 with both) attended control clinics and 3311 (2437 with asthma and 1371 with COPD, including 497 with both) attended intervention clinics (figure 2, table 1). Two-thirds (67\%) of participants were women, and the mean age at start of follow-up was 51.4 (SD 18.3) years. Participants with asthma were less likely than those with COPD to be men $(29 \%$ vs $44 \%)$ and tended to be younger (mean ages 47.8 and 62.6 years, respectively) (table 1). These participants were identified among all 72324 adults who attended control clinics and 81750 who attended intervention clinics during the same period. All participants were included in the assessment of trial outcomes, either at individual level (for participants with asthma or COPD) or at clinic level (for assessment of rates of new asthma and COPD diagnoses among all attendees).

The baseline characteristics of participants with asthma or COPD, or both, in intervention and control clinics were compared if they attended a participating clinic during either baseline 1 or baseline 2 periods (table 1, figure 2). Participants newly diagnosed during the follow-up period are excluded from these results because these data were mostly not available or applicable to them. During both baseline periods, participants with asthma and those with COPD in intervention clinics were slightly less likely to be men and had slightly higher mean ages than those in control clinics. They were also slightly more likely to have ever received prescriptions for ICS, SABA and LABA+ICS. Participants with asthma in intervention clinics were slightly more likely than those in control clinics to have treatment started or changed or spirometry requested during the baseline periods, resulting in higher asthma scores; these differences were greater during baseline 1 than during baseline 2. Participants with COPD had the same pattern of differences between intervention and control clinics in treatments and spirometry at baseline, resulting in higher COPD scores in intervention clinics. Differences in COPD scores were larger during baseline 1 than during baseline 2. Management of comorbid cardiovascular disease, diabetes and depression was similar in intervention and control clinics. Rates of new diagnoses of asthma and COPD were similar in intervention and control clinics during baseline 1 but were slightly lower in intervention clinics during baseline 2. Hospital admission rates were similar in intervention and control clinics.

Trial outcomes during the 12 months of follow-up are shown in table 2. Among participants with asthma, those in intervention clinics were more likely to have a start or change in treatment $(19.0 \%$ vs $15.1 \%, \mathrm{p}=0.012)$ and to have spirometry requested $(11.0 \%$ vs $8.1 \%, \mathrm{p}=0.012)$, resulting in higher asthma score $(\mathrm{p}=0.006)$. Among participants with COPD, those in intervention clinics were not more likely to have a start or change in treatment $(10.7 \%$ vs $10.1 \%, \mathrm{p}=0.699)$ or to have spirometry requested $(16.1 \%$ vs $13.0 \%, \mathrm{p}=0.053)$, resulting in no difference in COPD score ( $\mathrm{p}=0.142)$. Among participants with asthma or COPD, or both, there were no differences in management of comorbidities or tobacco cessation medication, which, with the exception of cardiovascular risk assessment, were all less than $4 \%$. Rates of new diagnoses of asthma and COPD, and of hospital admissions, did not differ between intervention and control clinics.

The magnitude of effects of the intervention on trial outcomes were estimated with and without adjustment for clinic level mean baseline values of the corresponding outcome variable (table 3 ). The OR of having a higher asthma score was 1.32 (95\% confidence limit (CL) 1.08 to $1.61, \mathrm{p}=0.006$ ) in intervention clinics compared with control clinics, without adjustment for baseline. The corresponding adjusted OR was 1.15 (0.94 to 1.41 , $\mathrm{p}=0.173$ ) with adjustment for baseline values recorded in each clinic during baseline 1, and 1.24 (95\% CL 1.03 to $1.50, \mathrm{p}=0.022$ ) with adjustment for baseline values recorded in each clinic during baseline 2. ORs for the two components of the asthma score (treatment and spirometry) were similar to those for the composite asthma score, except that the OR for start or change of treatment remained statistically significant at the 5\% level with adjustment for baseline values recorded during baseline 1 (OR 1.21, 95\% CL 1.01 to $1.45, \mathrm{p}=0.041$ ). In participants with COPD, ORs were slightly higher than one for all but one of the outcomes, but none of these was statistically significant at the $5 \%$ level. Management of comorbid cardiovascular disease, diabetes and depression, prescriptions for tobacco cessation, and rates of new asthma and COPD diagnosis, and of hospital admissions, did not differ between intervention and control clinics, 
Table 1 Baseline characteristics of participants* in intervention and control arms

\begin{tabular}{|c|c|c|c|c|c|c|c|c|}
\hline & \multicolumn{4}{|c|}{$\begin{array}{l}\text { Baseline } 1 \\
\text { (before follow-up began) }\end{array}$} & \multicolumn{4}{|c|}{$\begin{array}{l}\text { Baseline } 2 \\
\text { (before training began) }\end{array}$} \\
\hline & \multicolumn{2}{|c|}{ Control } & \multicolumn{2}{|c|}{ Intervention } & \multicolumn{2}{|c|}{ Control } & \multicolumn{2}{|c|}{ Intervention } \\
\hline & $\mathbf{N}$ & $\%$ & $\mathbf{N}$ & $\%$ & $\mathbf{N}$ & $\%$ & $\mathbf{N}$ & $\%$ \\
\hline Participants with asthma & 2206 & 100.0 & 2017 & 100.0 & 2161 & 100.0 & 1918 & 100.0 \\
\hline Sex (male) & 614 & 27.8 & 515 & 25.5 & 591 & 27.4 & 485 & 25.3 \\
\hline Inhaled corticosteroid ever & 820 & 37.2 & 814 & 40.4 & 752 & 34.8 & 703 & 36.7 \\
\hline Short acting beta ${ }_{2}$-agonist ever & 761 & 34.5 & 755 & 37.4 & 705 & 32.6 & 652 & 34.0 \\
\hline Long acting beta ${ }_{2}$-agonist+ICS ever & 302 & 13.7 & 320 & 15.9 & 269 & 12.5 & 275 & 14.3 \\
\hline Any of the above ever prescribed & 995 & 45.1 & 1005 & 49.8 & 911 & 42.2 & 883 & 46.0 \\
\hline$\dagger$ Asthma score $=0$ & 1782 & 80.8 & 1502 & 74.5 & 1748 & 80.9 & 1483 & 77.3 \\
\hline Asthma score $=1$ & 366 & 16.6 & 428 & 21.2 & 348 & 16.1 & 369 & 19.2 \\
\hline Asthma score=2 & 58 & 2.6 & 87 & 4.3 & 65 & 3.0 & 66 & 3.4 \\
\hline Asthma score: start or change treatmentł & 339 & 15.4 & 387 & 19.2 & 319 & 14.8 & 333 & 17.4 \\
\hline Asthma score: spirometry $\ddagger$ & 143 & 6.5 & 215 & 10.7 & 159 & 7.4 & 168 & 8.8 \\
\hline \multirow[t]{2}{*}{ COPD ever diagnosed } & 442 & 20.0 & 468 & 20.2 & 437 & 20.2 & 453 & 23.6 \\
\hline & Mean & SD & Mean & SD & Mean & SD & Mean & SD \\
\hline Clinic level asthma score & 0.214 & 0.107 & 0.293 & 0.124 & 0.206 & 0.110 & 0.287 & 0.119 \\
\hline \multirow[t]{2}{*}{ Age (years) } & 48.3 & 18.0 & 49.1 & 17.9 & 48.0 & 18.8 & 48.7 & 17.8 \\
\hline & $\mathrm{N}$ & $\%$ & $\mathrm{~N}$ & $\%$ & $\mathrm{~N}$ & $\%$ & $\mathrm{~N}$ & $\%$ \\
\hline Participants with COPD & 1042 & 100.0 & 1190 & 100.0 & 1025 & 100.0 & 1169 & 100.0 \\
\hline Sex (male) & 450 & 43.2 & 493 & 40.7 & 445 & 43.4 & 471 & 40.3 \\
\hline Inhaled corticosteroid ever & 343 & 32.9 & 390 & 32.2 & 306 & 29.9 & 342 & 29.5 \\
\hline Short acting beta ${ }_{2}$-agonist ever & 302 & 29.0 & 341 & 32.2 & 274 & 26.7 & 299 & 25.6 \\
\hline Long acting beta ${ }_{2}$-agonist+ICS ever & 213 & 20.9 & 264 & 28.1 & 188 & 18.3 & 223 & 19.1 \\
\hline Any of the above ever prescribed & 463 & 44.4 & 549 & 45.3 & 417 & 40.7 & 482 & 41.2 \\
\hline COPD score $=0 \dagger$ & 88.1 & 84.6 & 943 & 77.8 & 849 & 82.8 & 948 & 81.1 \\
\hline COPD score $=1$ & 130 & 12.5 & 220 & 18.2 & 145 & 14.2 & 175 & 15.0 \\
\hline COPD score $=2$ & 31 & 3.0 & 48 & 4.0 & 31 & 3.0 & 46 & 4.9 \\
\hline COPD score: start or change treatmentł & 93 & 8.9 & 137 & 11.3 & 95 & 9.3 & 125 & 10.7 \\
\hline COPD score: spirometry $\ddagger$ & 99 & 9.5 & 181 & 14.9 & 112 & 10.9 & 142 & 12.2 \\
\hline \multirow[t]{2}{*}{ Asthma ever diagnosed } & 442 & 42.4 & 468 & 38.6 & 437 & 42.6 & 453 & 38.8 \\
\hline & Mean & SD & Mean & SD & Mean & SD & Mean & SD \\
\hline \multirow[t]{2}{*}{ Age (mean) } & 62.2 & 13.4 & 61.9 & 13.4 & 61.7 & 13.1 & 61.1 & 13.6 \\
\hline & $\mathrm{N}$ & $\%$ & $\mathrm{~N}$ & $\%$ & $\mathrm{~N}$ & $\%$ & N & $\%$ \\
\hline Participants with asthma or COPD & 2806 & 100.0 & 2761 & 100.0 & 2749 & 100.0 & 2632 & 100.0 \\
\hline Sex (male) & 885 & 31.5 & 840 & 30.4 & 857 & 31.2 & 794 & 30.1 \\
\hline Cardiovascular disease newly diagnosed§ & 91 & 3.2 & 124 & 4.5 & 1147 & 4.2 & 130 & 4.9 \\
\hline Cardiovascular risk assessed $\emptyset$ & 1785 & 63.6 & 1751 & 63.4 & 1829 & 66.5 & 1791 & 68.0 \\
\hline Diabetes newly diagnosed & 91 & 3.2 & 113 & 4.1 & 86 & 3.1 & 97 & 3.7 \\
\hline Nicotine or buproprion or nortriptyline prescribed & 76 & 2.7 & 102 & 3.7 & 87 & 3.2 & 90 & 3.4 \\
\hline Depression newly diagnosed & 69 & 2.5 & 107 & 3.9 & 65 & 2.4 & 80 & 3.0 \\
\hline \multirow[t]{2}{*}{ Antidepressant treatment started } & 87 & 3.1 & 118 & 4.3 & 105 & 3.8 & 117 & 4.4 \\
\hline & Mean & SD & Mean & SD & Mean & SD & Mean & SD \\
\hline Clinic level COPD score & 0.186 & 0.078 & 0.269 & 0.173 & 0.202 & 0.834 & 0.225 & 0.102 \\
\hline Age (years) & 51.3 & 18.0 & 52.7 & 17.8 & 51.1 & 17.8 & 52.2 & 17.6 \\
\hline
\end{tabular}


Table 1 Continued

\section{Baseline 1 \\ Baseline 2 \\ (before follow-up began) \\ (before training began)}

\begin{tabular}{|c|c|c|c|c|c|c|c|c|}
\hline New asthma and COPD diagnoses & 10.3 & 4.2 & 10.9 & 4.7 & 11.2 & 4.3 & 9.5 & 3.1 \\
\hline New asthma diagnoses & 7.3 & 3.2 & 7.4 & 3.9 & 7.8 & 3.7 & 6.2 & 2.7 \\
\hline New COPD diagnoses & 3.0 & 1.9 & 3.6 & 2.2 & 3.4 & 1.4 & 3.3 & 1.4 \\
\hline Hospital admissions for asthma or COPD & 1.3 & 1.1 & 0.80 & 0.61 & 0.57 & 0.65 & 0.59 & 0.46 \\
\hline
\end{tabular}

*Excludes participants newly diagnosed with asthma or COPD during follow-up.

†Primary outcomes.

$\ddagger$ Components added to create primary outcomes.

§ICD-10 I00-I99.

ПBlood pressure, cholesterol,glucose and/or ECG recorded.

${ }^{*}$ No of new diagnoses or hospital admissions: 1000 adults visiting clinic during the year.

COPD, chronic obstructive pulmonary disease; ICS, inhaled corticosteroid.

with or without adjustment for baselines. Effect estimates did not differ between subgroups with newly or previously diagnosed asthma or COPD, or between subgroups with both asthma and COPD and those with only one condition ( $p>0.05$ for interaction terms) (results not shown).

\section{DISCUSSION}

The trial indicates that educational outreach training of primary care doctors and nurses to use the PACK guide for the management of respiratory diseases, compared with provision of the guide without training, was associated with small increases in initiation or change of treatment and use of spirometry for adults diagnosed with asthma. Conservatively using a $1.7 \%$ significance level, to account for having three primary outcomes, the difference in asthma score remains statistically significant $(p=0.006)$. Similar patterns of outcomes and ORs were seen for participants with COPD, but these associations were not statistically significant. The intervention was not shown to affect the rate of new diagnoses of asthma or COPD, prescriptions for tobacco cessation, or the management of comorbid cardiovascular disease, diabetes or depression, all of which were very low with the exception of cardiovascular risk assessment which included blood pressure measurement.

When estimated effects on the primary endpoints were adjusted to account for clinical activity during baseline 1 (which included training for at least 8 months), the magnitude of these effects was reduced, and estimated effects on primary outcomes became non-significant. The latter adjustment, proposed in the original protocol, ${ }^{18}$ might however be excessive if, as the results suggest, training had begun to have effects during baseline one period (table 1). However, the OR for start or change of treatment in participants with asthma-the main component of the asthma score-was consistent despite either adjustment and remained statistically significant.

Interpretation of these results requires several considerations. The trial evaluated only the training component of the programme, and not the other components (PACK guide, health system strengthening and monitoring and evaluation), which were provided to both control and intervention clinics. However, access to the guide may indeed have influenced clinical practice in control clinics, as suggested by the $60 \%$ increase $(0.330 / 0.206)$ in mean asthma scores and the $39 \%$ increase $(0.281 / 0.202)$ in mean COPD scores, in control clinics from baseline 2 (table 1) to the follow-up period (table 2). These changes in control clinics reduced the trial's scope for demonstrating additional improvements in intervention clinics due to training alone.

We have used this approach to evaluating the impact of PACK training in our previous trials with other versions of PACK, with small, consistent and generally positive results. ${ }^{23-26}$ The current trial demonstrates small but significant effects on improving management of at least one endpoint. As we have previously argued, pragmatic trials, with their potential for contamination despite cluster randomisation, unplanned co-interventions and other factors, when negative are of little help to health planners (other than in ruling out harm) but, when positive, provide compelling evidence for adopting a complex intervention, especially if the new method is well received by providers and recipients of care. ${ }^{27}$

The selection of participants and data collection methods need to be considered. Compared with our prior calculations, the actual sample sizes and statistical power were greater, but the differences in primary outcomes were smaller than anticipated. In contrast to our previous trials in low-income and middle-income countries, the relatively high rates of previous treatment among participants before training began (table 1) may have limited the scope for training to further increase initiation and changes of treatment. Almost half of participants with asthma and over $40 \%$ with COPD had already received some treatment, and over $40 \%$ of both groups had already received ICS, either alone or in combination with a LABA. These treatment rates are similar to those reported in surveys from Brazil as a whole but are still not optimal. ${ }^{28} 29$ In asthma, use of 
Table 2 Follow-up values of outcome variables in intervention and control clinics

\begin{tabular}{|c|c|c|c|c|c|c|}
\hline \multirow[b]{2}{*}{ Individual level outcomes } & \multicolumn{2}{|c|}{ Control } & \multicolumn{2}{|c|}{ Intervention } & \multirow[t]{2}{*}{$P$ value } & \multirow[t]{2}{*}{ ICC } \\
\hline & $\mathbf{N}$ & $\%$ & $\mathbf{N}$ & $\%$ & & \\
\hline Participants with asthma & 2633 & 100.0 & 2437 & 100.0 & & \\
\hline${ }^{*}$ Asthma score $=0$ & 2107 & 80.0 & 1823 & 74.8 & $0.006 \dagger$ & 0.028 \\
\hline Asthma score $=1$ & 442 & 16.8 & 497 & 20.4 & & \\
\hline Asthma score $=2$ & 84 & 3.2 & 117 & 4.8 & & \\
\hline Asthma score component: spirometry & 212 & 8.1 & 267 & 11.0 & 0.012 & 0.017 \\
\hline $\begin{array}{l}\text { Asthma score component: start or change of } \\
\text { treatment, including: }\end{array}$ & 398 & 15.1 & 464 & 19.0 & 0.012 & 0.020 \\
\hline ICS started & 156 & 5.9 & 178 & 7.3 & 0.067 & 0.039 \\
\hline Long-acting beta $_{2}$-agonist+ICS started & 63 & 2.4 & 85 & 3.5 & 0.083 & 0.067 \\
\hline Treatment stepped up & 30 & 1.1 & 43 & 1.8 & 0.096 & 0.074 \\
\hline Treatment stepped down & 22 & 0.8 & 20 & 0.8 & 0.932 & 0.023 \\
\hline Participants with COPD & 1181 & 100.0 & 1371 & 100.0 & & \\
\hline *COPD score $=0$ & 951 & 80.5 & 1065 & 77.7 & 0.142 & 0.036 \\
\hline COPD score $=1$ & 186 & 15.8 & 245 & 17.9 & & \\
\hline COPD score $=2$ & 44 & 3.7 & 61 & 4.5 & & \\
\hline COPD score: spirometry & 154 & 13.0 & 220 & 16.1 & 0.053 & 0.034 \\
\hline COPD score: start or change treatment, including: & 120 & 10.2 & 147 & 10.7 & 0.699 & 0.013 \\
\hline ICS started & 61 & 5.2 & 65 & 4.7 & 0.513 & 0.006 \\
\hline Short-acting beta ${ }_{2}$-agonist & 51 & 4.3 & 62 & 4.5 & 0.880 & 0.005 \\
\hline Long-acting beta ${ }_{2}$-agonist+ICS started & 63 & 5.3 & 83 & 6.1 & 0.456 & 0.018 \\
\hline Participants with asthma or COPD & 3355 & 100.0 & 3311 & 100.0 & & \\
\hline Cardiovascular disease newly diagnosed & 107 & 3.2 & 119 & 3.6 & 0.705 & 0.005 \\
\hline Cardiovascular risk assessed & 2170 & 64.7 & 2141 & 64.7 & 0.698 & 0.034 \\
\hline Diabetes newly diagnosed & 66 & 2.0 & 96 & 2.9 & 0.057 & 0.009 \\
\hline Nicotine or buproprion or nortriptyline prescribed & 102 & 3.0 & 115 & 3.5 & 0.419 & 0.005 \\
\hline Depression newly diagnosed & 81 & 2.4 & 104 & 3.1 & 0.266 & 0.020 \\
\hline \multirow[t]{2}{*}{ Antidepressant treatment started } & 121 & 3.6 & 132 & 4.0 & 0.607 & 0.013 \\
\hline & Mean & SD & Mean & SD & $P \ddagger$ & \\
\hline Clinic level asthma score & 0.330 & 0.142 & 0.361 & 0.131 & $0.456 \ddagger$ & Not applicable \\
\hline Clinic level COPD score & 0.281 & 0.175 & 0.293 & 0.150 & $0.808 \ddagger$ & Not applicable \\
\hline \multicolumn{7}{|c|}{ Clinic level diagnosis and hospital admission ratios§ } \\
\hline "New asthma and COPD diagnoses & 9.3 & 3.0 & 9.4 & 4.0 & $0.884 \ddagger$ & Not applicable \\
\hline New asthma diagnoses & 6.2 & 1.8 & 6.0 & 2.6 & $0.706 \ddagger$ & Not applicable \\
\hline New COPD diagnoses & 3.1 & 1.9 & 3.3 & 2.1 & $0.851 \ddagger$ & Not applicable \\
\hline Hospital admissions for asthma or COPD & 1.1 & 1.2 & 1.1 & 0.0 & $0.822 \ddagger$ & Not applicable \\
\hline
\end{tabular}

*Primary outcomes.

†Ordinal logistic regression model. Logistic regression models for all other individual-level outcomes. All individual-level regression models adjusted for randomisation strata and intra-cluster correlation of outcomes.

łLinear regression models adjusted for randomisation strata.

$\S$ No of new diagnoses or hospital admissions: 1000 adults visiting clinic during the year.

COPD, chronic obstructive pulmonary disease; ICC, intra-cluster correlation coefficient; ICS, inhaled corticosteroid.

ICS and other controllers should be higher and so the increase in prescribing in the present study, although small, is gratifying. But for COPD, as Zimmermann Teixeira and colleagues report, the more common problem in Brazil is overtreatment with ICS for mildly severe
COPD and undertreatment for severe disease. ${ }^{29}$ Thus, the relatively high use of ICS in primary care patients observed in Florianópolis, who might have milder disease compared with others receiving specialist care, may still provide room for improvement. 


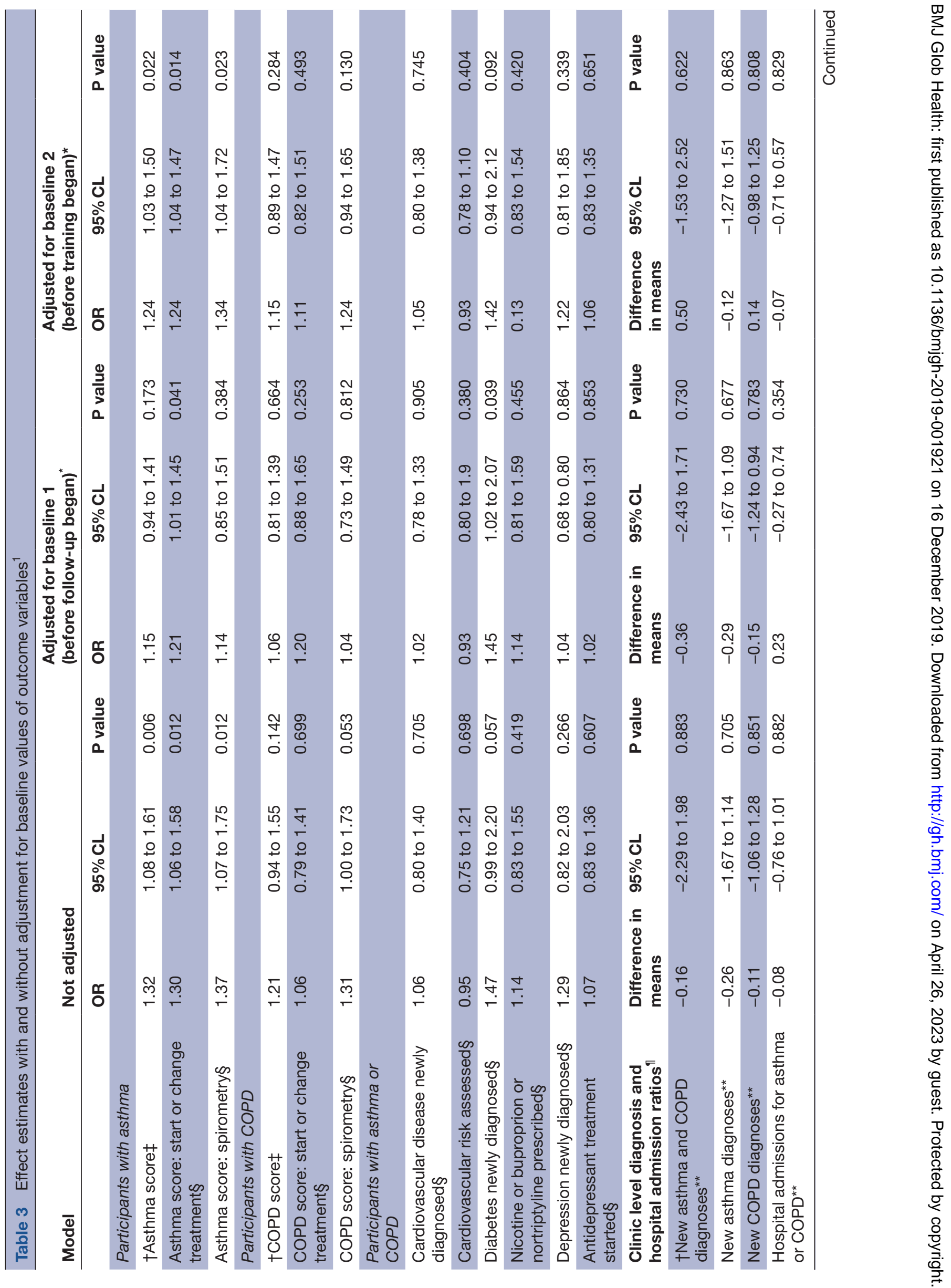


Our use of routine electronic medical records to identify and characterise eligible participants may have led to inclusion of some participants to whom trial outcomes were not relevant. Some may have been diagnosed with asthma or COPD years earlier but asymptomatic or well controlled during the trial, and thus did not need the investigations or treatments used as trial outcomes. We included participants newly diagnosed with asthma or COPD during the follow-up period because they would presumably be most in need of investigation or new treatments. Electronic medical records did not permit us to accurately assess participants' respiratory health status, as respiratory symptom questionnaires and spirometry would have done, to identify individuals who most needed to start or change treatment or to evaluate health outcomes. Instead, we relied on process indicators of effective and improved management.

Other trial design issues relate to the nature and intensity of training. PACK training is performed on-site and in-service, and is continuous, being conducted in short sessions at fortnightly intervals. Since the guide is comprehensive and covers the the most common conditions managed in primary care facilities, it is possible that, within the trial's training period, there was insufficient emphasis on the management of respiratory conditions to produce larger effects. The follow-up period might also be too short to detect changes in clinical practice. We will therefore continue to monitor outcomes after completion of this trial, for delayed effects, or changes in control clinics after they receive training.

This study setting had favourable features. Florianópolis is a leader in Brazil both in its primary care provision and in its electronic medical record system which provides considerable scope for evaluating practice innovations such as PACK. Electronic medical records enabled us to characterise clinical practices and identify and include all eligible patients who used municipal clinics in the entire city, thus enhancing generalisability and providing a large sample size at minimal cost. This follows our successful use of electronic medical records in randomised trials of PACK's precursors in South Africa. ${ }^{24} 25$

This study contributes to implementation research on methods for improving primary respiratory care. The results support the growing evidence of the effectiveness of the training component of the PACK approach in which clinicians are introduced to customised, up-todate, evidence-based, point-of-care clinical decision support. ${ }^{23-26}$ Thus, although the impact of training is not large nor consistent across asthma and COPD, within the context of the global implementation of PACK as a learning health system, the results add evidence that the training intervention is effective on a different continent and in a different socioeconomic and health system context. This trial does not explain how PACK works, or what element is most effective, but supports qualitative and quantitative evidence previously reported in more than 50 peer-reviewed publications. ${ }^{23} 2430$ A qualitative process evaluation is under way. Early findings 
indicate that trainers, trainees and health officials were enthusiastic about PACK and that training stimulated a desire for continuing professional development. Attendance at training sessions was high, but staff turnover meant that supplementary training was needed for new staff. Training was felt to improve cooperation between doctors and nurses, while expanding nurses roles' and confidence. Political changes in local government, and fiscal constraints arising from Brazil's economic crisis, did not affect implementation during the study. The results of this trial are consistent with the most recent and comprehensive review of healthcare provider strategies to improve performance which advocates for combining training with group problem solving and supervision, both of which are built into the PACK implementation approach. ${ }^{31}$

The results support expanding nurses' roles and strengthening nurse-doctor co-operation in asthma care. Nurses were not authorised to prescribed asthma or COPD medication or to request spirometry, but they were trained to refer patients whom they identified as needing these interventions to doctors in their primary care teams. Training doctors and nurses together aimed to align their clinical decision-making. These results add to previous evidence about interventions to promote interprofessional collaboration. ${ }^{32} 33$

In conclusion, this study shows that it was feasible to localise and implement PACK in municipal primary care services across a Brazilian city, and that PACK training led to improvements in asthma care within a year of completing initial training.

\section{Author affiliations}

${ }^{1}$ Norwich Medical School, University of East Anglia Faculty of Medicine and Health Sciences, Norwich, UK

${ }^{2}$ Knowledge Translation Unit, University of Cape Town Lung Institute, Cape Town, Western Cape, South Africa

${ }^{3}$ University of Sao Paulo, Sao Paulo, Brazil

${ }^{4}$ ProAR, Universidade Federal da Bahia, Salvador, Bahia, Brazil

${ }^{5}$ Gerência de Integração Assistencial, Secretaria Municipal de Saúde, Florianópolis, Santa Catarina, Brazil

${ }^{6}$ LIHS, University of Leeds, Leeds, UK

${ }^{7}$ Public Health, Universidade Federal de Santa Catarina, Florianópolis, Santa

Catarina, Brazil

${ }^{8}$ Biostatistics Unit, Medical Research Council, Cape Town, South Africa

Twitter Sonia Natal @sonianatal

Acknowledgements The authors are grateful for the Florianópolis City Health Department for permission to carry out the study, to the doctors and nurses in clinics who participated in the training and using of the PACK guide; to Dr Tracy Eastman (Director of PACK Global Development at Knowledge Translation Unit and BMJ Publishing Group) for support with agreements, project management and quality assurance. This work was supported by the Florianópolis City Health Department which funded in-country localisation of PACK Adult, printing of the PACK guide, training and clinical support. Use of PACK Global Adult guide and associated materials was provided free of charge to Florianópolis City Health Department by BMJ Publishing Group on behalf of the Knowledge Translation Unit. Mentoring of the localisation of the guide and training materials by the Knowledge Translation Unit was funded by the Peter Sowerby Foundation (http://www.pete rsowerbyfoundation.com/) as part of development of a localisation toolkit for PACK.

Contributors MOB, EDB, RS, AAC, MPdA, RZ, JZ, SN, CL and LRF conceived of and designed the study. EDB, MPdA, RZ, JZ, SN, RVC, CW, LA, DG-P and LRF contributed to development of the PACK Brasil Adulto—versão Florianópolis guide and training materials. MPdA collated the electronic medical record data and MOB carried out the statistical analysis. All authors contributed to writing the paper and approved the final manuscript.

Funding The authors have not declared a specific grant for this research from any funding agency in the public, commercial or not-for-profit sectors.

Competing interests We have read and understood BMJ policy on declaration of interests and declare that CW, LRF, DG-P, LA, RVC and EDB are employees of the KTU. RZ and MPdA are employees of the City Health Department, Florianópolis, Brazil. JZ is an ex-employee of the City Health Department, Florianópolis, Brazil and a current holder of full-time $\mathrm{PhD}$ studentship from the Brazilian research agency CNPq. AAC reports grants, personal fees and non-financial support from GSK, personal fees and non-financial support from AstraZeneca, personal fees and non-financial support from Boehringer Ingelheim, personal fees and non-financial support from MEDA, personal fees and non-financial support from Novartis, personal fees and non-financial support from EUROFARMA, personal fees and non-financial support from CHIESI, personal fees and non-financial support from Boston Scientific, outside the submitted work. EDB reports grants to institution for clinical trials from Novartis, Boehringer Ingelheim, Merck, Takeda, GlaxoSmithKline, Hoffmann La Roche, Actelion, Chiesi, Sanofi-Aventis, Cephalon, TEVA and AstraZeneca and personal fees from ICON, Novartis, Cipla, Vectura, Cipla, Menarini, ALK, ICON, Sanofi Regeneron, Boehringer Ingelheim and AstraZeneca, outside the submitted work, and is a Member of Global Initiative for Asthma Board and Science Committee. RS reports grants for clinical trials, personal, institution fees and nonfinancial support from AstraZeneca, and personal fees and non-financial support from Novartis, Boehringer Ingelheim, CHIESI and EUROFARMA. Since August 2015, the KTU and BMJ have been engaged in a non-profit strategic partnership to provide continuous evidence updates for PACK, expand PACK-related supported services to countries and organisations as requested, and where appropriate license PACK content. The KTU and BMJ co-fund core positions, including a PACK Global Development Director, and receive no profits from the partnership. PACK receives no funding from the pharmaceutical industry. This paper forms part of a Collection on PACK sponsored by the BMJ to profile the contribution of PACK across several countries towards the realisation of comprehensive primary health care as envisaged in the Declarations of Alma Ata and Astana.

Patient consent for publication Not required.

Ethics approval The study was approved by the research ethics committee of the Federal University of Santa Catarina.

Provenance and peer review Not commissioned; externally peer reviewed.

Data availability statement № data are available.

Open access This is an open access article distributed in accordance with the Creative Commons Attribution Non Commercial (CC BY-NC 4.0) license, which permits others to distribute, remix, adapt, build upon this work non-commercially, and license their derivative works on different terms, provided the original work is properly cited, appropriate credit is given, any changes made indicated, and the use is non-commercial. See: http://creativecommons.org/licenses/by-nc/4.0/.

\section{ORCID iDs}

Max Oscar Bachmann http://orcid.org/0000-0003-1770-3506

Alvaro A Cruz http://orcid.org/0000-0002-7403-3871

Camilla Wattrus http://orcid.org/0000-0002-2075-7903

\section{REFERENCES}

1 GBD 2016 Brazil Collaborators. Burden of disease in Brazil, 1990-2016: a systematic subnational analysis for the Global Burden of Disease Study 2016. Lancet 2018;392:760-75.

2 Casas A, Montes de Oca M, Menezes AM, et al. Respiratory medication used in COPD patients from seven Latin American countries: the LASSYC study. Int J Chron Obstruct Pulmon Dis 2018;13:1545-56.

3 To T, Stanojevic S, Moores G, et al. Global asthma prevalence in adults: findings from the cross-sectional World Health Survey. BMC Public Health 2012;12.

4 Rzewuska M, de Azevedo-Marques JM, Coxon D, et al. Epidemiology of multimorbidity within the Brazilian adult general population: evidence from the 2013 National Health Survey (PNS 2013). PLoS One 2017;12:e0171813.

5 Massuda A, Hone T, Leles FAG, et al. The Brazilian health system at crossroads: progress, crisis and resilience. BMJ Glob Health 2018;3:e000829. 
6 Ponte EV, Cruz AA, Athanazio R, et al. Urbanization is associated with increased asthma morbidity and mortality in Brazil. Clin Respir J 2018;12:410-7.

7 Ponte EV, Cruz Álvaro A., Lima VB, et al. Greater access to long acting beta2 agonists is associated with less hospital admissions due to COPD: a longitudinal nation-wide study. Lung 2018;196:643-8.

8 Cerci Neto A, Ferreira Filho OF, Bueno T, et al. Reduction in the number of asthma-related hospital admissions after the implementation of a multidisciplinary asthma control program in the city of Londrina, Brazil. J Bras Pneumol 2008;34:639-45.

9 Souza-Machado C, Souza-Machado A, Franco R, et al. Rapid reduction in hospitalisations after an intervention to manage severe asthma. Eur Respir J 2010;35:515-21.

10 Cornick R, Wattrus C, Eastman T, et al. Crossing borders: the pack experience of spreading a complex health system intervention across low-income and middle-income countries. BMJ Glob Health 2018;3.

11 Fairall L, Cornick R, Bateman E. Empowering frontline providers to deliver universal primary healthcare using the practical and approach to care kit. BMJ Glob Health 2018;3.

12 Cornick R, Picken S, Wattrus C, et al. The practical approach to care kit (PACK) guide: developing a clinical decision support tool to simplify, standardise and strengthen primary healthcare delivery. BMJ Glob Health 2018;3:e000962.

13 Simelane ML, Georgeu-Pepper D, Ras C-J, et al. The practical approach to care kit (PACK) training programme: scaling up and sustaining support for health workers to improve primary care. BMJ Glob Health 2018;3:e001124.

14 Awotiwon A, Sword C, Eastman T, et al. Using a mentorship model to localise the practical approach to care kit (PACK): from South Africa to Nigeria. BMJ Glob Health 2018;3:e001079.

15 Mekonnen Y, Hanlon C, Emyu S, et al. Using a mentorship model to localise the practical approach to care kit (PACK): from South Africa to Ethiopia. BMJ Glob Health 2018;3:e001108.

16 Tsima BM, Setlhare V, Nkomazana O. Developing the Botswana primary care guideline: an integrated, symptom-based primary care guideline for the adult patient in a resource-limited setting. $J$ Multidiscip Healthc 2016;9:347-54.

17 Wattrus C, Zepeda J, Cornick RV, et al. Using a mentorship model to localise the practical approach to care kit (PACK): from South Africa to Brazil. BMJ Glob Health. In Press 2018;3.

18 Bachmann MO, Bateman ED, Stelmach R, et al. Integrating primary care of chronic respiratory disease, cardiovascular disease and diabetes in Brazil: practical approach to care kit (PACK Brazil): study protocol for randomised controlled trials. J Thorac Dis 2018;10:4667-77.

19 Global Initiative for Asthma (GINA). Global strategy for asthma management and prevention, 2018. Available: https:ginasthma. orgwp-contentuploads201804wms-GINA-2018-report-tracked_v1.3. pdf [Accessed 30 May 2019].

20 Vogelmeier CF, Criner GJ, Martinez FJ, et al. Global strategy for the diagnosis, management, and prevention of chronic obstructive lung disease 2017 report: gold executive summary. Am J Respir Crit Care Med 2017;195:557-82.

21 Medical Research Council. Cluster randomised trialsmethodological and ethical considerations, 2002. Available: https: www.cebma.orgwp-contentuploadsCluster-randomised-trialsMethodological-and-ethical-considerations.pdf [Accessed 30 May 2019].

22 Haines A, Ashcroft R, Coggon D, et al. Personal information in medical research, 2000. Available: https:www.mrc.ac.ukdocume ntspdfpersonal-information-in-medical-research [Accessed 30 May 2019].

23 Fairall LR, Zwarenstein M, Bateman ED, et al. Effect of educational outreach to nurses on tuberculosis case detection and primary care of respiratory illness: pragmatic cluster randomised controlled trial. BMJ 2005;331:750-4

24 Zwarenstein M, Fairall LR, Lombard C, et al. Outreach education for integration of HIV/AIDS care, antiretroviral treatment, and tuberculosis care in primary care clinics in South Africa: PALSA plus pragmatic cluster randomised trial. BMJ 2011;342:d2022.

25 Fairall L, Bachmann MO, Lombard C, et al. Task shifting of antiretroviral treatment from doctors to primary-care nurses in South Africa (STRETCH): a pragmatic, parallel, cluster-randomised trial. Lancet 2012;380:889-98.

26 Fairall LR, Folb N, Timmerman V, et al. Educational outreach with an integrated clinical tool for nurse-led non-communicable chronic disease management in primary care in South Africa: a pragmatic cluster randomised controlled trial. PLoS Med 2016;13:e1002178.

27 Fairall LR, Mahomed O, Bateman ED. Evidence-based decisionmaking for primary care: the interpretation and role of pragmatic trials. S Afr Med J 2017;107:278-9.

28 Maspero JF, Jardim JR, Aranda A, et al. Insights, attitudes, and perceptions about asthma and its treatment: findings from a multinational survey of patients from Latin America. World Allergy Organ J 2013;6.

29 Zimermann Teixeira PJ, Ferreira Nogueira M. COPD: more treatment will translate to better breathing. Will it? J Bras Pneumol2019;45.

30 Knowledge Translation Unit. Publications, 2019. Available: https: knowledgetranslation.co.zaresearchpublications [Accessed 30 May 2019]

31 Rowe AK, Rowe SY, Peters DH, et al. Effectiveness of strategies to improve health-care provider practices in low-income and middle-income countries: a systematic review. Lancet Glob Health 2018;6:e1163-75.

32 Wranik WD, Price S, Haydt SM, et al. Implications of interprofessional primary care team characteristics for health services and patient health outcomes: a systematic review with narrative synthesis. Health Policy 2019;123:550-63.

33 Reeves S, Pelone F, Harrison R, et al. Interprofessional collaboration to improve professional practice and healthcare outcomes. Cochrane Database Syst Rev;19. 